\title{
距irestice
}

\section{Quarta geração das tecnologias de apoio ao idoso para ambientes de vida assistida}

Fourth generation of technologies to support the elderly for assisted living environments

Siony da Silva ${ }^{1}$, Josceli Maria Tenório², Ivan Torres Pisa ${ }^{3}$

\section{Resumo}

Objetivo: O objetivo desta revisão é identificar os recursos tecnológicos que estão sendo utilizados no acompanhamento de vida dos idosos. Método: Foi realizada pesquisa de literatura de artigos publicados entre janeiro de 2013 a dezembro de 2017 na base Pubmed. Resultados: Foram selecionados artigos que descrevem recursos tecnológicos como apoio nas atividades diárias dos idosos e os recursos tecnológicos utilizados em Ambientes de Vida Assistida (Ambient Assisted Living AAL). Os artigos foram selecionados com o objetivo de conhecer os recursos que estão sendo empregados, as vantagens e as limitações decorrentes de sua implementação. Identificou-se as gerações de AAL. Conclusão: Constatou-se que as pesquisas sobre a implantação das tecnologias no acompanhamento de idosos ainda estão sendo realizadas em caráter experimental. Pela análise do material identificou-se que vários recursos tecnológicos podem ser empregados, configurando diferentes gerações de AAL. A partir dessa análise foi elaborada uma proposta de definição da Quarta Geração de ALL preconize interoperabilidade entre os dispositivos tecnológicos e na utilização de tecnologias que colaborem com a qualidade de vida e interação social do idoso.

Palavras-Chave: Idoso. Ambiente de Vida Assistida. Tecnologias.

\begin{abstract}
Objective: The objective of the analysis is the technological resources that are being used to monitor the lives of the elderly. Method: A literature review was conducted in the Pubmed database considering 2013 january to 2017 december period. Results: Technological resources were treated as with the support of the daily tools of the elderly and the technological resources used in the Ambient Assisted Living (AAL). The pages were selected with the purpose of knowing the resources that are being used, as well as the advantages and the restrictions of its implementation. It was identified as Generations of AAL. Conclusion: It was verified that the researches on the implantation of the technologies in the accompaniment of the elderly are being carried out in an experimental character. Through the analysis of the material it was observed that several technological resources can be employed, configuring in Assisted Living Environment generations. Based on this analysis, the proposal of the Fourth Generation was elaborated in such a way that interoperability occurs between the technological devices, and that the use of technologies can collaborate with the quality of life and social interaction of the elderly.

1 Mestre. Instituto Federal de Educação, Ciência e Tecnologia de São Paulo. Email: sionysilva@gmail.com 2 Doutoranda. Programa de Pós Graduação em Gestão e Informática em Saúde - Universidade Federal de São Paulo (UNIFESP). Email: josceli2006@hotmail.com

3 Doutor. Departamento de Informática em Saúde - Universidade Federal de São Paulo. Email: ivanpisa@gmail.com Correspondência: Dep. de Informática em Saúde (UNIFESP) - Rua Botucatu, 862 - Edifício José Leal Prado - térreo. Vila Clementino, São Paulo, SP - Brasil. CEP: 04023-062.
\end{abstract}


Keywords: Elderly. Ambient Assisted Living. Technology.

\section{Introdução}

O avanço dos recursos tecnológicos aliado ao acesso à informação, à prevenção e ao tratamento de doenças têm aumentado a expectativa de vida da população. O envelhecimento pode ser considerado um êxito para as políticas de saúde pública e para o desenvolvimento socioeconômico, mas também se constitui um desafio. De fato, é preciso que a sociedade se adapte para melhorar 0 sistema de saúde, a capacidade funcional, a participação social e a segurança dos idosos, respeitando sua individualidade e independência. À medida em que a pessoa envelhece aumentam os fatores de vulnerabilidade provocados por processos fisiológicos, como a diminuição da visão ou o esquecimento, e por fatores sociais, como viver sozinho, depressão e usar medicamentos psicoativos ${ }^{1}$.

Este cenário tem estimulado pesquisas para elucidar necessidades e riscos das atividades diárias dos idosos ${ }^{1}$. Ambientes de vida assistida (Ambient Assisted Living AAL) são sistemas considerados essenciais para melhorar os cuidados de idosos para que possam manter a autonomia e independência ${ }^{2}$ geralmente baseados em uso de sensores e de tecnologias de comunicação cujo objetivo é colaborar com a saúde e qualidade de vida das pessoas ${ }^{3}$.

Este artigo apresenta uma revisão sobre a geração das tecnologias utilizadas no acompanhamento de idosos em suas residências e propõe uma quarta geração dos AAL.

\section{Métodos}

Este estudo foi baseado na revisão bibliográfica de estudos publicados de janeiro de 2013 a dezembro de 2017 na base

Pubmed (https://www.ncbi.nlm.nih.gov/pubmed).

Foram selecionados artigos originais em português, inglês e espanhol que descreveram recursos tecnológicos como apoio nas atividades diárias dos idosos. Os termos para recuperação de artigos foram: ambient assisted living, technology and aging e smart home.

Foram selecionados artigos que estivessem relacionados ao tema por meio da leitura do título, resumo e texto completo. Outros artigos foram inseridos por conveniência por serem considerados relevantes ao assunto.

\section{Resultados}

A longevidade é um fenômeno mundial. O processo do envelhecimento é contínuo, pessoal, progressivo e dinâmico e envolve aspectos sociais econômicos e 
biológicos. Ao longo do tempo, tem sido desenvolvidas tecnologias com o objetivo de acompanhar os idosos em sua vida diária. Tais recursos podem ser simples ou com alto grau de tecnologia. Entre estes recursos, as tecnologias de vida assistida têm o potencial de acompanhar os idosos para que possam manter independência e autonomia em suas atividades. Tais tecnologias devem ser acessíveis, eficientes e/ou de alta qualidade. Devem possibilitar vida autônoma, manter a privacidade dos dados pessoais, favorecer a inclusão social e possibilitar uma vida com dignidade ${ }^{3}$. Em situações específicas podem oferecer recursos de prevenção de saúde. Ferramentas baseadas nessas tecnologias podem ser utilizadas pela própria pessoa, por profissionais de saúde ou por pessoas que acompanham o paciente/usuário.

A denominação Ambiente de Vida Assistida representa um campo multidisciplinar que envolve a pesquisa e a aplicação de tecnologia da informação e comunicação, das ciências sociais e de pesquisa médica. Visa desenvolver sistemas pessoais de saúde e telessaúde para acompanhar os efeitos do envelhecimento. São, portanto, sistemas personalizados, adaptados às necessidades do usuário e que integram alta qualidade médica, conforto, interoperabilidade, usabilidade, segurança e precisão. Isto envolve desafios para que sejam adotados em larga escala. Normalmente, sistemas de AAL oferecem suporte para idosos na prevenção (monitoramento e prevenção de quedas), compensação e suporte (tarefas de apoio cognitivo) e envelhecimento ativo e independente 4 .

A maioria dos sistemas de AAL explora as atividades que estão sendo realizadas pelos idosos em sua residência e visa avaliar ou inferir no estado funcional de idosos. As alterações na rotina podem auxiliar na detecção precoce de doenças.

Considerando as características de AAL, foram propostas três gerações para o acompanhamento do idoso ${ }^{5}$. São consideradas tecnologias de primeira geração, aquelas cujos recursos são acionados pelo idoso em caso de emergência. Consiste no uso de um telefone ou de um dispositivo eletrônico que é acionado pelo usuário. Uma central telefônica, por exemplo, realiza o contato com o idoso e determina se um serviço de emergência e se os cuidadores serão ou não necessários. Sistemas de primeira geração são geralmente simples, confiáveis e reduzem o estresse do usuário e da família. A limitação desses dispositivos consiste que o usuário esteja com ou próximo do dispositivo. Além disso, o usuário não pode estar incapacitado física ou mentalmente para acioná-lo em uma emergência ${ }^{6}$.

Nas tecnologias de segunda geração são empregados sensores que têm a capacidade de detectar sinais de emergência sem que o usuário tenha que acionar algum dispositivo. Apesar dos 
benefícios do emprego desses recursos usuários podem considerá-los invasivos por seu aspecto mais autônomo ${ }^{6}$.

As tecnologias de terceira geração consideram o uso de recursos que além de detectarem os sinais, também relatam e evitam problemas ao empregar os conceitos mais amplos de ambiente de vida assistida. Esses sistemas monitoram o ambiente, a mobilidade, os sinais vitais e atividades da vida diária do idoso ${ }^{6}$.

Os dispositivos empregados para o acompanhamento do idoso podem ser classificados em dispositivos de alarme (primeira geração); dispositivos de monitoramento de vida diária e monitoramento de sinais vitais. Os dois últimos podem ser identificados na segunda ou terceira geração em função de características de integração, mobilidade e ubiquidade da proposta de implantação.

Diferentes combinações de recursos podem ser utilizados para prevenir os riscos dos idosos em sua residência, entre eles sensor de vibração no chão e vídeo (detectar queda); sensor acoplado na caixa de medicação (verificar se o usuário tem tomado o medicamento no horário da prescrição); detectores de fumaça e alarme (risco de incêndio); detectores de monóxido de carbono (intoxicação por gás) e propostas para estimular a atividade física e social (gamificação, exergaming) ${ }^{1}$.

É possível acompanhar o estado de saúde do idoso por meio da medição do fluxo de água no banheiro e na cozinha, por exemplo. As atividades monitoradas podem ser a micção, o trabalho na cozinha e as atividades relacionadas a manutenção de higiene pessoal. É uma proposta de baixo custo que se baseia em sensores de fluxo de água e de movimento ${ }^{7}$.

Embora a implantação de AAL seja de grande importância para a sociedade, pesquisas $^{8}$ destacam que os sistemas não apresentam requisitos para personalização e adaptação. Também apontam a dificuldade na implantação devido à baixa interoperabilidade entre os sistemas ${ }^{8}$.

Em geral os idosos querem viver de forma independente em suas casas, sendo que a independência é influenciada pelo estado de saúde, idade e gênero. $O$ conceito de independência envolve poder cuidar de si mesmo sem depender dos outros; fazer o que tem vontade e não se sentir obrigado a alguém. As evidências sobre a eficácia de casas inteligentes para permitir a independência de idosos ainda são escassas. Os idosos que não fazem uso de casa inteligente avaliam que poderiam utilizá-la embora expressem preocupações conceituais em seu uso. Além disso, acreditam que tais recursos possam ser adequados para outras pessoas e não para si mesmos. A implantação de projetos deve considerar essas opiniões e os desenvolvedores de tecnologias, os cuidadores e as decisões políticas precisam incorporar uma visão ampla sobre a independência do indivíduo. Ainda, a noção de independência pode 
variar de pessoa para pessoa e ao longo do tempo 9 .

A adoção de tecnologias para idosos envolve fatores relacionados ao desenho físico, características individuais que afetam a interação com as tecnologias, aspectos sociais como cultura e expectativa do idoso no uso do recurso e a forma de comunicação e uso dos recursos. A adoção das tecnologias deve considerar os seguintes fatores: valor (percepção de utilidade e potencial benefício); usabilidade (percepção de facilidade de uso e aprendizagem); custo (altos custos de tecnologia afastam os idosos de utilizálas); marketing sobre 0 uso das tecnologias, ou seja, as pessoas precisam ter informações sobre os recursos que podem utilizar; suporte técnico no uso dos recursos; suporte social (apoio social e de parentes também influenciam a utilização da tecnologia); capacidade técnica e benefícios afetivos devem ser considerados na proposta de tecnologias; independência (percepção de se sentir independente e de que o uso de recursos tecnológicos não sejam estigmatizantes); experiência (importância da experiência passada no uso das tecnologias) e confiança no uso dos recursos ${ }^{10}$.

As perspectivas para os próximos 25 anos consideram 0 uso de sistemas integrados aos registros dos pacientes e o emprego de maior quantidade de sensores não invasivos. Haverá um movimento contrário à utilização dos recursos tecnológicos, o que representará uma resistência individual e familiar. Os modelos serão cada vez mais personalizados e as casas serão equipadas com sistema inteligente (iluminação, temperatura, segurança etc.) facilitando a incorporação dos projetos. Destaca-se para o futuro a relevância de se definir e implantar padrões de interoperabilidade e segurança de dados para os ambientes de vida assistida ${ }^{3}$.

Vale lembrar que vários fatores influenciam o envelhecimento, tais como os “...biológicos, psicológicos, sociais, comportamentais, econômicos, culturais e ambientais", havendo, portanto, "heterogeneidade no processo de envelhecer"11 sendo fundamental que propostas de AAL considerem as características individuais do usuário.

Os benefícios de sua incorporação são representados na Figura 1, assim como as limitações relacionadas à interoperabilidade dos sistemas, aspectos éticos e transição de protótipos para ambientes reais.

A mudança na estrutura familiar, o aumento da expectativa de vida, doenças crônicas e degenerativas que podem acometer as pessoas e a necessidade de acompanhamento personalizado com redução de custos são elementos que contribuem para a implantação de AAL.

Incorporar tecnologias no acompanhamento de idosos possibilita benefícios significativos, mas é preciso analisar a estrutura tecnológica em que será desenvolvida, os fatores psicológicos 
e sociais que a implantação poderá proporcionar e atentar para as necessidades reais do usuário final. Se o usuário - o próprio idoso e seus familiares, responsáveis ou cuidadores - não aceitarem tais recursos, todo o processo estará fadado ao insucesso. A velhice ainda é vista em termos negativos como sinônimo de doença e deficiência, motivo pelo qual muitos estudos focam o emprego das tecnologias da informação e comunicação como recursos de monitoramento de idosos nesta situação. Este estereótipo contrasta com o alto grau de heterogeneidade da população idosa ${ }^{12}$.

Embora pesquisas estejam sendo realizadas para implantar ambientes de vida assistida, existe dificuldade em passar do protótipo para o ambiente de vida real porque torna-se necessário um domínio técnico da tecnologia utilizada além de contemplar as necessidades específicas de cada idoso ${ }^{8}$. Existem desafios em relação aos requisitos de aceitabilidade, durabilidade, facilidade, comunicação e energia dos dispositivos vestíveis

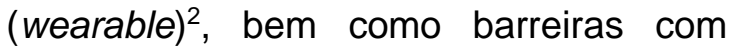
relação ao uso das tecnologias ${ }^{1}$.

A maioria dos estudos sobre ambientes de vida assistida não demonstra evidências clínicas satisfatórias sobre a qualidade de vida alcançada pela introdução desses projetos ${ }^{2}$. O nível de aceitação dos usuários em relação à usabilidade e questões éticas e de privacidade não são explorados em detalhes. Também há limitações quanto à incorporação das necessidades de usuários e cuidadores e nem sempre os cuidadores e profissionais de saúde são bem orientados sobre o emprego do sistema. O potencial do uso de sensores, bem como armazenamento e acesso distribuído da informação (por exemplo, em nuvem) ainda não foi apreciado nos trabalhos. Considera-se necessário melhorar a identificação dinâmica de padrões irregulares dos sinais para 0 reconhecimento da atividade de um idoso. Por outro lado, há evidências de que o emprego das tecnologias amplia a possibilidade do idoso continuar vivendo em sua residência, reduz sentimentos de solidão, depressão e vulnerabilidade, previne riscos e deterioração da condição de saúde ${ }^{13}$.

Além disso, agiliza o atendimento do paciente, amplia a adesão ao tratamento e dependendo da proposta de acompanhamento minimiza internações e problemas decorrentes das atividades diárias. Existem projetos de acompanhamento de vida diária do idoso que utilizam a integração de sensores e pesquisas com a chamada internet das coisas (IoT), mas essas pesquisas ainda estão em fase inicial ${ }^{14}$.

Considerando a evolução das tecnologias, a Figura 2 apresenta uma proposta de definição da Quarta Geração de AAL baseada nas três gerações anteriores ${ }^{6}$.

$\mathrm{Na}$ proposta da quarta geração, ocorre uma integração e interoperabilidade 
entre diversos dispositivos de informação e comunicação, levantamento de dados ambientais e dispositivos wearables de forma transparente para o usuário (IoT). Este acompanhamento possibilita maior qualidade de vida porque minimiza eventuais problemas que poderão ocorrer com o idoso. Outro fator que poderá colaborar para a qualidade de vida é o fato de que as tecnologias ampliam a interação social e familiar, isto porque existe uma preocupação de muitos pesquisadores em desenvolver ambientes amigáveis que atendam às normas de usabilidade.

O idoso poderá participar diretamente da implementação dos ambientes personalizados. Isto gerará confiança no uso do AAL, em especial relacionado à privacidade que poderá ser controlada pelo próprio usuário dependendo da condição física e cognitiva em que se encontrar. $O$ emprego de $A A L$ ampliará a necessidade de políticas públicas de incentivo e redução de custo, além de aumentar a pesquisa sobre sua utilização.

O aumento da expectativa de vida precisa ser acompanhado de propostas que consideram o sistema público de saúde, as tecnologias de acessibilidade e o treinamento dos profissionais de saúde. É preciso conscientização da sociedade em respeitar os idosos independente de suas capacidades ou limitações decorrentes de processos fisiológicos ou de enfermidade para que possam manter a qualidade de vida e sua autonomia.
Projetos educacionais devem fazer parte de políticas governamentais para que os idosos se sintam acolhidos em suas necessidades e não sofram reforço de estigmatização. Existem questões que precisam ser analisadas na implantação de ambientes de vida assistida na área técnica, mas também na área cultural e social.

Considerando o avanço das tecnologias, a longevidade da população mundial e a preocupação em desenvolver ambientes que atendam as necessidades individuais das pessoas idosas, novos recursos serão incorporados aos $A A L$ tornando estes ambientes com menor custo de implantação e manutenção, maior aderência do usuário, aumento na integração de profissionais e aumento da interoperabilidade dos dispositivos de tal forma que se possa transformar protótipos em experiências bem sucedidas para os idosos, profissionais de saúde, cuidadores, família e que tenham impacto comercial.

\section{Conclusão}

O envelhecimento da população está provocando mudanças na sociedade com significativa ampliação de pesquisas para que as pessoas possam permanecer por mais tempo em suas residências - no conceito ageing in place - com independência e segurança.

Várias tecnologias têm sido incorporadas em ambientes de vida assistida, tais como câmera de vídeo, 
microfones, detectores de presença, sensores de temperatura, luz, fumaça, umidade, sensores inerciais, sensores vestíveis etc.

A implantação de ambientes de vida assistida deve considerar diferentes elementos, entre eles a necessidade do usuário, custo, segurança dos recursos, interoperabilidade, aceitação do usuário e em muitas situações do cuidador e ou da família.

É essencial que atendam aos aspectos de segurança, custos, efetividade, impacto social, ético e legal, aspectos logísticos e eficiência. É também fundamental a definição de uma equipe multidisciplinar que analise as necessidades individuais do idoso e realize uma proposta para atendê-lo de forma que ele não sinta invasão de privacidade e consequentemente aceite a tecnologia como um recurso válido para sua vida.

Projetos estão sendo desenvolvidos internacionalmente. Várias pesquisas realizam protótipos do emprego desses recursos sem que ocorra a implantação efetiva para que possa ser realizada uma análise mais detalhada sobre os benefícios e limitações nas rotinas diárias, bem como a aceitação de uso pelos usuários. Ainda há e possivelmente sempre haverá um grupo de idosos sem acesso aos recursos tecnológicos e que pode ter alguma resistência e dificuldade em utilizá-la. Ao mesmo tempo é preciso elaborar estudos para prover recursos para a próxima geração de idosos cujo acesso à tecnologia fez parte de sua vida.

Políticas públicas devem contemplar a pesquisa, e a capacitação de equipes multidisciplinares.

Este artigo explora as possibilidades da Quarta Geração de AAL, ambiente em que ocorre a interoperabilidade entre os diversos recursos tecnológicos, a facilidade de uso, segurança dos dados e privacidade do usuário, a possibilidade de interação social e familiar, elementos que ampliam a qualidade de vida e favorecem o ageing in place.

\section{Referências}

1.Yared R, Abdulrazak B. (2016).

Ambient Technology to Assist Elderly People in Indoor Risks. J Computers, vol. 5. Disponível em:

http://www.mdpi.com/2073-

$431 \mathrm{X} / 5 / 4 / 22 / \mathrm{htm}$

2.Al-Shaqi R, Mourshed M, Rezgui Y. Progress in ambient assisted systems for independent living by the elderly. SpringerPlus, 2016; 5:624. Disponível em: http://doi.org/10.1186/s40064-016-2272-8

3.Haux R, Koch S, Lovell N, Marschollek M, Nakashima N, Wolf K. (2016). HealthEnabling and Ambient Assistive Technologies: Past, Present, Future, IMIA Yearbook of Medical Informatics.

Disponível em:

https://www.researchgate.net/publication/3 04661754_Health-

Enabling_and_Ambient_Assistive_Techno logies_Past_Present_Future

4.Dobre C, Constandinos XM, Garcia NM, Mastorakis G, Rossitza IG. (2017). Introduction to the AAL and ELE Systems. Disponível em: 
https://doi.org/10.1016/B978-0-12805195-5.00001-6.

5. Doughty K, Cameron K, Garner P.(1996). Three generations of telecare of the elderly, J. Telemed. Telecare 2, 7180. Disponível em: 10.1177 / 1357633 X9600200202.

6. Blackman S, Matlo C, Bobrovitskiy C, Waldock $A$, Fang $M L$, Jackson PJ, Milahidis A, Nygard L, Astell A, Sixsmith A. (2015). Ambient

Assisted Living Technologies for Aging Well: A Scoping Review. Journal of Intelligent Systems, 25(1), pp. 55-69. doi:10.1515/jisys-2014-0136.

7. Tsukiyama T. (2015). In-home Health Monitoring System for Solitary Elderly. Procedia Computer Sciense, vol 23. https://www.sciencedirect.com/science/arti cle/pii/S1877050915024734.

8. Memon M, Wagner SR, Pedersen CF, Beevi FHA, Hansen FO. (2014). Ambient Assisted Living Healthcare Frameworks, Platforms, Standards, and Quality Attributes. Sensors (Basel, Switzerland), 14(3), 4312-4341. Disponível em: http://doi.org/10.3390/s140304312.

9.Peek STM, Aarts S, Wouters E. (2015). Can Smart Home Technology Deliver on the Promise of Independent Living? A Critical Reflection Based on the Perspectives of Older Adults. 1-10. 10.1007/978-3-319-01904-8_41-1.

10. Lee C, Coughin JF. (2014).

Perspective: Older Adults' Adoption of Technology: An Integrated Approach to Identifying Determinants and Barriers. Journal of Product Innovation Management Vol 32. , Ed.. 5 , 747-759. Disponível em:

https://www.researchgate.net/publication/2 62808824_PERSPECTIVE_Older_Adults \%27_Adoption_of_Technology_An_Integr ated_Approach_to_Identifying_Determina nts_and_Barriers

11.Katzenstein T, Schwartz G, Almeida MHM. (2012). Reflexões sobre aproximação de idosos a tecnologias de informação e comunicação a partir dos arquétipos Senex* e Puer, São Paulo, SP: PUC-SP: Revista Kairós Gerontologia, 15(2), 203-218. Disponível em: https://revistas.pucsp.br/index.php/kairos/ article/view/9883.

12. Sixsmith A. (2013) Technology and the Challenge of Aging. In: Sixsmith A., Gutman G. (eds) Technologies for Active Aging. International Perspectives on Aging, vol 9. Springer, Boston, MA . Disponível em: https://link.springer.com/chapter/10.1007/ 978-1-4419-8348-0_2\#citeas.

13.Alexandru A, lancuslecu M. (2017). Enabling Assistive Technologies to Shape the Future of the Intensive Senior-Centred Care: A Case Study Approach. Studies in Informatics and Control 26 (3) 343-352, September. Disponível em: https://sic.ici.ro/wpcontent/uploads/2017/10/SIC_2017-3Art10.pdf

14. Azimi I, Rahmani AM, Liljeberg $P$, Tenhunen HC. Internet of things for remote elderly monitoring: a study from user-centered perspective. Journal of Ambient Intelligence and Humanized Computing April 2017, Volume 8, Issue 2, pp 273289

Disponível em:

https://link.springer.com/article/10.1007/s1 2652-016-0387-y. 


\section{Anexos}

\section{Lista de figuras}

Figura 1 - Mapa conceitual, consequências e limitações dos ambientes de vida assistida

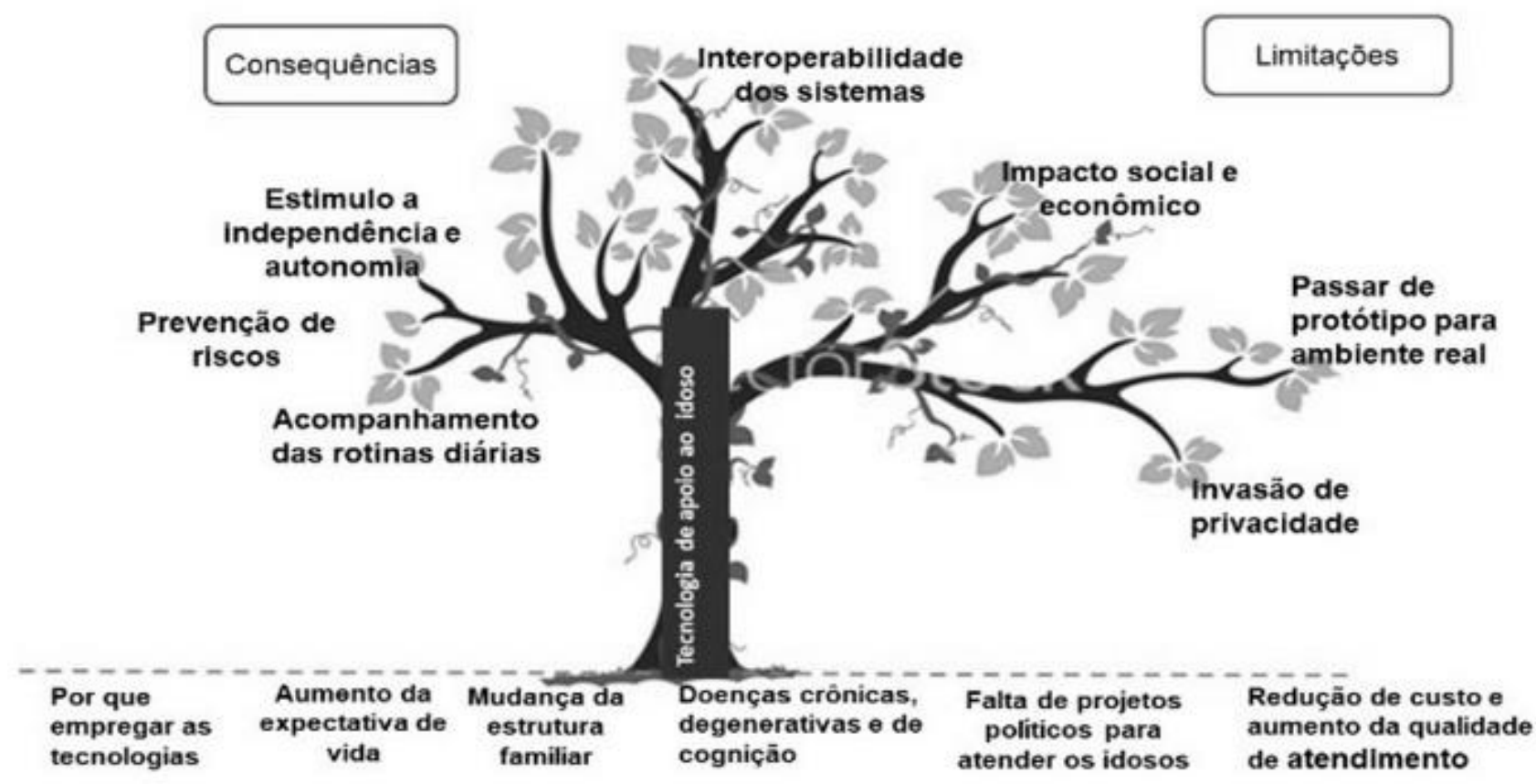

Fonte: os autores

Figura 2: Proposta de Quarta Geração de AAL

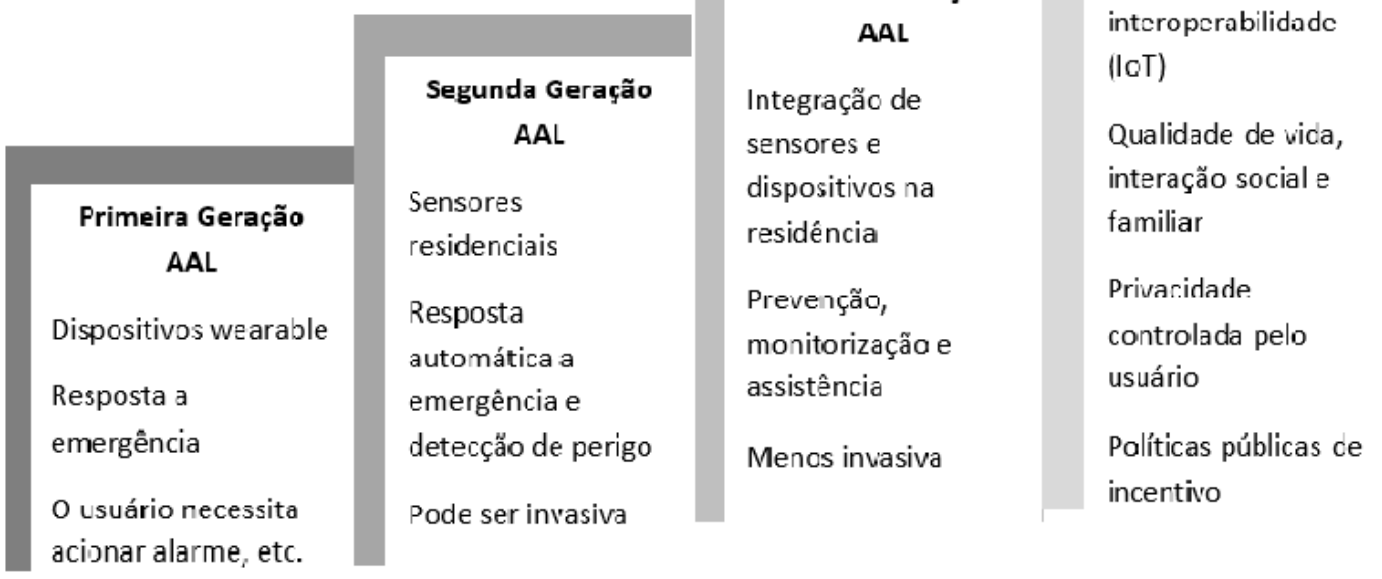

Fonte- Autores, baseada nas três gerações ${ }^{6}$ 


\section{Como citar este artigo}

Silva Sda, Tenório JM, Pisa IT. Quarta geração das tecnologias de apoio ao idoso para ambientes de vida assistida. Revista de Saúde Digital e Tecnologias Educacionais. [online], volume 4, n. 1. Editor responsável: Luiz Roberto de Oliveira. Fortaleza, mês e ano, p. 74-84. Disponível em: http://periodicos.ufc.br/resdite/index. Acesso em "dia/mês/ano".

Data de recebimento do artigo: 20/05/2019

Data de aprovação do artigo: 18/06/2019 